BULL. AUSTRAL. MATH. SOC.

VOL. 32 (1985), 415-418.

\title{
DERIVATIONS ON COMMUTATIVE OPERATOR ALGEBRAS
}

\author{
Mark Spivack
}

It is well-known that any derivation on a commutative von Neumann algebra is implemented by a bounded operator. In this note we present a simple alternative proof, which generalises the result further within Hilbert space, and to reflexive Banach spaces.

It is well known that any derivation on a commutative von Neumann algebra is implemented by a bounded operator. This result can be extended to certain non-self-adjoint commutative algebras by similarity. In this note we present a simple alternative proof, which generalises the result further within Hilbert space, and to reflexive Banach spaces.

Let us call a set of Banach space operators a projection algebra if it is the algebra, or norm-closed algebra, generated by its projections. We will say that a projection algebra is bounded, with bound $k$, if the real number $k$ is a uniform bound for the projections in the algebra. If $E$ is a Banach space, then $B(E)$ denotes the set of bounded operators on $E$ and a derivation on a subalgebra $A$ is a linear map $\delta$ from $A$ ințo $B(E)$ such that $\delta(a b)=a \delta(b)+\delta(a) b$ for $a, b$ in $A$. A derivation is said to be implemented if it is of the form $d_{x}(a)=x a-a x$ for some fixed operator $x$ on $E$ and all $a$ in $A$. All Banach spaces mentioned are assumed to be complex, and all algebras to be unital. Note that any "complete" Boolean algebra of projections is bounded. (See [2]).

Every derivation on a commutative algebra generated by a finite set of Banach space projections is known to be implemented by an operator

Received 11 April 1985.

Copyright Clearance Centre, Inc. Serial-fee code: 0004-9727/85 $\$ A 2.00+0.00$. 
which can be described explicitly. We obtain a bound for this operator which is independent of the number of generating projections. It follows immediately that continuous derivations on commutative bounded projection algebras on reflexive Banach spaces are implemented.

We abuse notation a little by saying that projections $p$ and $q$ are orthogonal if $p q=q p=0$. Denote $1-p$ by $p^{\perp}$.

Then, given any finite commutative set $\left\{q_{i}\right\}, i=1, \ldots, n$ of projections, there is a set $\left\{p_{i}\right\}, i=1, \ldots, 2^{n}$, of orthogonal projections (some of which may be zero) generating the same algebra as $\left\{q_{i}\right\}$.

Before proving the crucial result we mention the fact that a Banach space $E$ is reflexive if and only if the unit ball of $B(E)$ is compact in the weak operator topology (Chapter VI, Dunford and Schwartz [2]). Note also that a von Neumann algebra on a Hilbert space is generated by its self-adjoint projections [1].

We first state as a lemma the known finite case.

LEMMA 1. Let $A$ be a finitely generated conmutative projection algebra on any Banach space $E$ and let $\delta$ be any derivation on $A$. If $\left\{p_{i}\right\}, i=1, \ldots, n$, is an orthogonal generating set, then the operator $x=\sum_{i=1}^{n} \delta\left(p_{i}\right) p_{i}$ implements $\delta$ on $A$.

The proof follows easily from the relations

$$
p \delta(p) p=0 \quad \text { and } \quad p \delta(q)=-\delta(p) q
$$

for orthogonal projections $p, q$.

THEOREM 2. $\|x\| \leqslant 4 k^{2}\|\delta\|$ where $k$ is a bound for $A$. If $E$ is a Hilbert space and the projections $\left\{p_{i}\right\}$ are self-adjoint, then $\|x\| \leqslant\|\delta\|$

Proof. We claim that for some integer $m$ we can write $x=\frac{1}{m} \sum_{r=1}^{m} \delta\left(u_{p}\right) u_{r}$, where for each $r,\left\|u_{r}\right\| \leqslant 2 k$. In fact we will put $u_{r}=\sum_{i=1}^{n} \lambda_{i, r} p_{i}$ where each $\lambda_{i, r}$ is 1 or -1 . 
Put $m=2^{n-1}$, and let $\left\{\lambda_{i, r}\right\}$ form the (finite) matrix

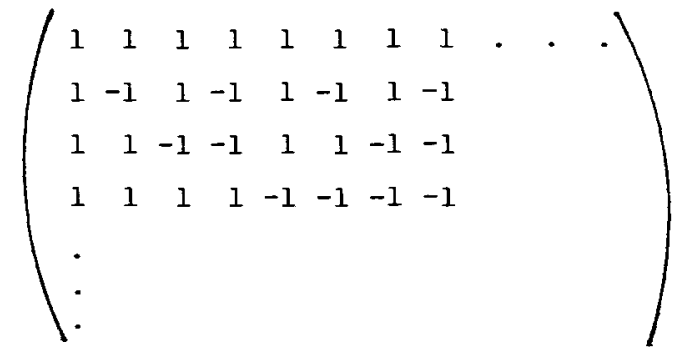

One can easily convince oneself, by checking with $n=2$ or 3 , that

$$
\sum_{x=1}^{m} \lambda_{i, r_{j, r}}=0 \text { for } i \neq j \text {. }
$$

and $\sum_{r=1}^{m}\left(\lambda_{i, r}\right)^{2}=m$ for each $i$. Consequently,

$$
\begin{aligned}
\sum_{r=1}^{m} \delta\left(u_{r}\right) u_{r} & =\sum_{r=1}^{m} \sum_{i, j=1}^{n} \lambda_{i, r} r_{j, r} \delta\left(p_{i}\right) p_{j} \\
& =\sum_{r=1}^{m} \sum_{i=1}^{n} \delta\left(p_{i}\right) p_{i}=m x \text {, as required. }
\end{aligned}
$$

Finally, since each $\lambda_{i, r}$ is $\pm l, u_{r}$ is the difference of two projections in $A$ and $u_{p}^{2}=1$. Hence $\left\|u_{r}\right\| \leqslant 2 k$, and $\|x\| \leqslant(2 k)^{2}\|\delta\|$. If $\left\{p_{i}\right\}$ is a set of self-adjoint Hilbert space projections then each $u_{r}$ is a self-adjoint unitary operator, and so $\left\|u_{p}\right\|=1$. Then $\|x\| \leqslant\|\delta\|$ as claimed.

We can immediately state the corollary.

COROLLARY 3. Let $A$ be any commutative bounded projection algebra on a reflexive Banach space $E$, and let $\delta$ be a continuous derivation on $A$. Then $\delta$ is implemented by an operator $x$ such that $\|x\| \leqslant 4 k^{2}\|\delta\|$, where $k$ is a bound for $A$. If, in particular, $E$ is a Hilbert space and $A$ a von Neumann algebra then we can choose $x$ so that $\|x\| \leqslant\|\delta\|$.

Proof. The restriction of $\delta$ to any finitely generated projection subalgebra $B$ is implemented by such an operator, by Theorem 2 . Let $S_{B}$ denote the set of operators which implement $\delta$ on $B$ and whose norms are bounded by $4 k^{2}\|\delta\|$. Then $S_{B}$ is compact in the weak operator topology, 
since $E$ is reflexive, and any finite intersection of such sets is nonempty. Hence the intersection of all such sets is non-empty, and the result follows.

REMARK 1. The condition on $E$ of reflexivity applies of course to Hilbext space, where the self-adjoint projections are exactly those of norm 1. Any projection on a Hilbert space which commutes with its adjoint must be self-adjoint. So a commutative projection algebra on a Hilbert space is self-adjoint if and only if it has bound 1 . Note that the result holds for general commutative projection $C^{*}$-algebras.

REMARK 2. We mention finally that this proof is closely related to an existing one for the case of a von Neumann algebra. I am indebted to Professor Erik Christensen for describing that one to me. We sketch it here for comparison: Let $\delta$ be a derivation on a commutative von Neumann algebra $A$. Note that by Ringrose [3] $\delta$ is continuous. Let $C$ be the weakly closed convex set generated by elements of the form $u^{*} \delta(u)$ where $u$ is unitary in $A$. For each unitary $u$ define a map $T_{u}: C \rightarrow C$ by $T_{u}(a)=u a u^{*}+u \delta\left(u^{*}\right)$. Then $C$ and the maps $T_{u}$ satisfy the conditions of the Markov-Kakutani fixed-point theorem [2], and so for some $x \in C$. $T_{u}(x)=x$ for all $u$. A simple manipulation shows that $x$ implements $\delta$. our proof is different even in this case in that it substitutes a partially constructive method for the use of the fixed-point theorem.

\section{References}

[1] J. Dixmier, Les algebres d'operateurs dans l'espace Hilbertien, 2nd edition (Gauthier-Villars, 1969).

[2] N. Dunford and J.T. Schwartz, Linear operators, (Interscience, 1958).

[3] J.R. Ringrose, "Automatic continuity of derivations on operator algebras", J. London Maths. Soc. 5 (1972), 432-438.

D.A.M.T.P.,

The University,

Silver street,

Cambridge,

England. 